\title{
Air traffic generation for new terminal area air traffic management concepts design and evaluation
}

\author{
Juan Besada, Iñigo Marquinez, Javier Portillo, \\ Gonzalo de Miguel and Ana Bernardos
}

\begin{abstract}
This paper describes two systems that can be used to obtain realistic random traffic samples in a terminal area: a real traffic analyser and a synthetic traffic generator. These two systems allow the air traffic management (ATM) engineer to gain insight on the traffic structure of the area under analysis, and allow obtaining realistic traffic samples enabling the evaluation of new operational concepts, the validation or system performance measurement after procedure changes, the analysis of ATM performance under forecasted future traffic changes, etc. Together with the design of the system, the work provides insight of user interfaces and describes the potential uses of such tools in an integrated ATM system.
\end{abstract}

\section{Keywords}

Air traffic management, air traffic analysis, air traffic simulation, air traffic modelling, traffic scenario definition

\section{Introduction}

Simulation is a basic technology for the design, evaluation, optimisation and training of different aspects of air traffic control and air traffic management. Historically, it has been widely used to design new operational concepts, including humans-in-the-loop simulations for validation (i.e. paired approaches, flight-deck-based merging ${ }^{2}$ and separation assurance ${ }^{3}$ ). Additionally, it has been used for the evaluation of system-wide performance 4 and for optimisation, using higher level modelling (as in oceanic airspace in the Pacific, ${ }^{5}$ in the National Airspace Systems, using multi-agent simulation ${ }^{6,7}$ ) or focusing at the node (airport or terminal area) - as in the Dulles Airport arrival operations redesign, ${ }^{8}$ in which Terminal Area Route Generation Evaluation and Traffic Simulation (TARGETS) software and Base of Aircraft Data (BADA) have been used. It is also typically used for airspace sectorisation, ${ }^{9}$ taking into account the maximum controller workload, and to design the allocation of traffic management to different controllers along the flight (such as route or terminal area ${ }^{10}$ ).

In particular, it is especially important to have realistic traffic simulations to design and validate new air traffic management (ATM) operational concepts with the advent of new ATM paradigms such as trajectorybased operations ${ }^{11}$ and the evolution of collaborative decision making, in which roles of pilot and controller are modified. ${ }^{12}$ The rapid changes appearing in Single
European Sky Advanced Research (SESAR) ${ }^{13}$ and NextGen ${ }^{14}$ projects make the simulations of special relevance.

An additional objective of simulation is the support to controller training. Some examples of this use may be found in early works related to the exploitation of artificial intelligence to ATC training ${ }^{15,16}$ or to the automatic tutoring of controller students. ${ }^{17}$ More modern descriptions of some of the aspects of those simulators are devoted to user interface description $^{18}$ such as an automatic speech recognition system enabling the simulation of voice communications with software agents simulating pilots or surrounding area controllers. ${ }^{19}$

A key problem of simulation is the quality of the models involved to reproduce the reality. Due to the enormous size of the complete ATM/ATC system it is impossible to work with extremely detailed models of all the involved agents, but, in order to be able to have accurate performance measures, it is very important to improve the current simple models. Examples of those improvements may be found in Gillet et al., ${ }^{20}$ 
where an improvement of aircraft behavioural model from historical radar data is described, or in Volf et al., ${ }^{21}$ where controller behaviour is modelled and validated against real data.

A problem that has received not so much attention in the literature is the automated generation of realistic traffic patterns, which is obviously a key input to any ATC simulator. In this paper, we describe two systems that can be used to obtain realistic traffic samples from a given real traffic database: a real traffic analyser and a synthetic traffic generator. The real traffic analyser obtains the patterns (statistical, determinist or mixed), to be further used as a possible input to the synthetic traffic generator block. These patterns summarise the overall traffic behaviour in a given controlled airspace area (terminal area, sector). For the expert, these patterns allow a deeper understanding of the overall space structure and of the actual operations being executed by controllers and pilots. Meanwhile, the synthetic traffic generator produces a synthetic traffic file with the generated aircraft features and associated flight plans, to be used in a realistic simulation to define traffic scenario under analysis. Those flights should be statistically consistent with the assumed traffic patterns. The paper will describe both systems in detail and their related human-machine interfaces (HMI), enabling a comprehensive analysis of the traffic under analysis and graphical validation of the generated traffic.

A similar effort, based in modifications of recorded data, is available in existing literature. ${ }^{22}$ In general, many of the operational systems for traffic scenario preparation are based on real traffic cloning or deletion performed by a human user. This is a very costly and error-prone method. The current prototype that has been developed for Indra under ATLANTIDA project. In this project a simulation facility similar to TARGETS, ${ }^{8,10}$ using BADA $^{23}$ prediction models, was developed for the study and validation of terminal area ATM concepts and automation. ${ }^{24}$ Based on our previous work, ${ }^{25}$ this paper includes a detailed description of the simulation system approach, the HMI and examples of the obtained results.

\section{High-level system description}

The main objective of the traffic analyser and generator is to produce a file with the information of the flight to be generated in a specific spatial distribution. It is important to note that the final output of the synthetic traffic generator is the necessary initial information (i.e. initial conditions and flight plans) to feed an ATM simulator. Figure 1 shows the main working blocks of the proposed systems. All data are described in XML files to ensure readability and processing capabilities.

The traffic analyser, taking the real flight information file and additional information, obtains the patterns (statistical, determinist or mixed), to be further used as an input to the synthetic traffic generator. The information input to this block is:

- Real traffic data: This is a file with the information of real flights for analysis. The data are recorded by the real-time flight data processing system, using the surveillance data to time-stamp the times of arrival to the airport, the overfly times of the entry points, etc.

- Operational context: These files contain a specification of the terminal area (TMA) physical structure: Entry points (Standard Arrivals STAR starting points), STARs (waypoint list), TMA exit points (Standard Instrument Departure SID ends and starting of overfly routes), SIDs (waypoint list), domestic routes, airports, etc.

- User preferences for the analysis: This configuration file contains: flags indicating whether the traffic

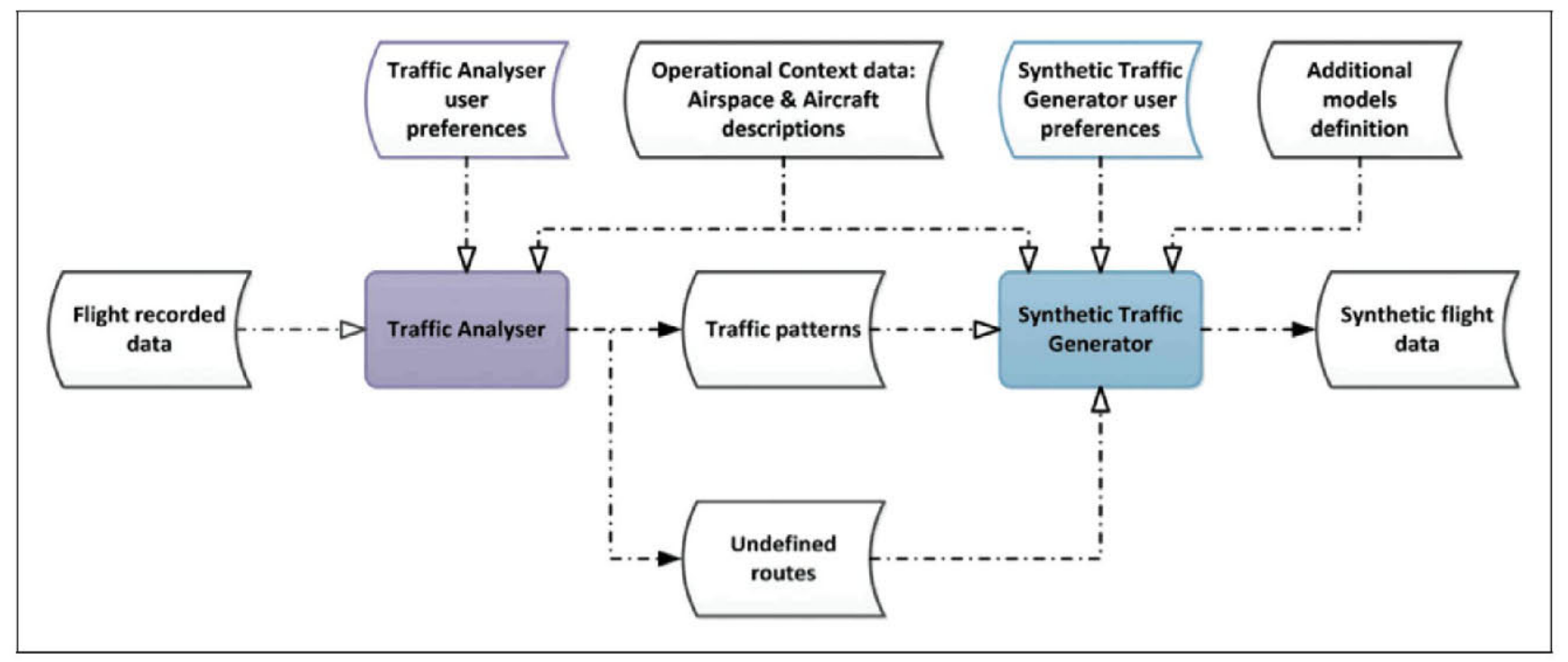

Figure I. Block diagram of the traffic analyser and synthetic traffic generator. 
analysis must obtain deterministic traffic patterns or not, and whether the traffic analysis must include the analysis of overfly flights; a set of thresholds used to define if the pattern under analysis has a deterministic form or not; a list of special days where the traffic does not follow the same pattern as others due to special circumstances (public holidays, long weekends...) and parameters of a polar grid used to group airports outside of the TMA with similar geographic location.

The information output of this block is:

- Traffic patterns.

- Undefined routes: A description of routes followed by traffic but not described in the operational context definition.

Meanwhile, the synthetic traffic generator (STG) generates the information about the initial conditions of the aircraft to be considered in the simulation exercise, as a starting point to the real traffic generation. The information input to this block is:

- Operational context: The same input as traffic analyser.

- Traffic patterns: A file provided by traffic analyser. The user may edit it to perform a controlled change of the traffic generation parameters and simulate their effect.

- Undefined routes: As provided by traffic analyser.

- Additional models to generate traffic: Some traffic features cannot be extracted from the real traffic file analysis and must be directly provided by the user.

- User preferences for the generation: Some additional parameters to be used at the generator: the time interval for generation; a list of special days; a set of traffic validation thresholds; the flight duration between injection time and arrival to TMA; and the minimum time separation at entry points.

The STG produces the synthetic traffic generated data: A file with the generated aircraft features and associated flight plans.

\section{Detailed functionality of traffic analyser}

This section details the functions comprising the traffic analyser. A basic assumption of the analysis is that an important part of the traffic in the TMA has a relevant deterministic component, as many of the arrival and departure flights will repeat on a periodic basis with the same flight plan. Superposed on that deterministic pattern there may exist a random pattern which will not follow such a predictable model. Therefore, our system considers the following traffic pattern analysis modes of operation:

- Mixed model. In this case, the traffic analyser extracts the parameters of a model with a deterministic and a random pattern. The deterministic pattern is extracted taking into account the repetitions of flights following periodic patterns (daily, weekly, monthly, and also regular special days, such as holiday start, end, etc.), by looking for repeated flight ID, origin, destination, etc. Once the deterministic flights are extracted, the random patterns are estimated from the remaining flights.

- Random model. In this case, the analyser treats the entire database file in order to obtain random traffic patterns and estimating their parameters, not searching for any deterministic pattern.

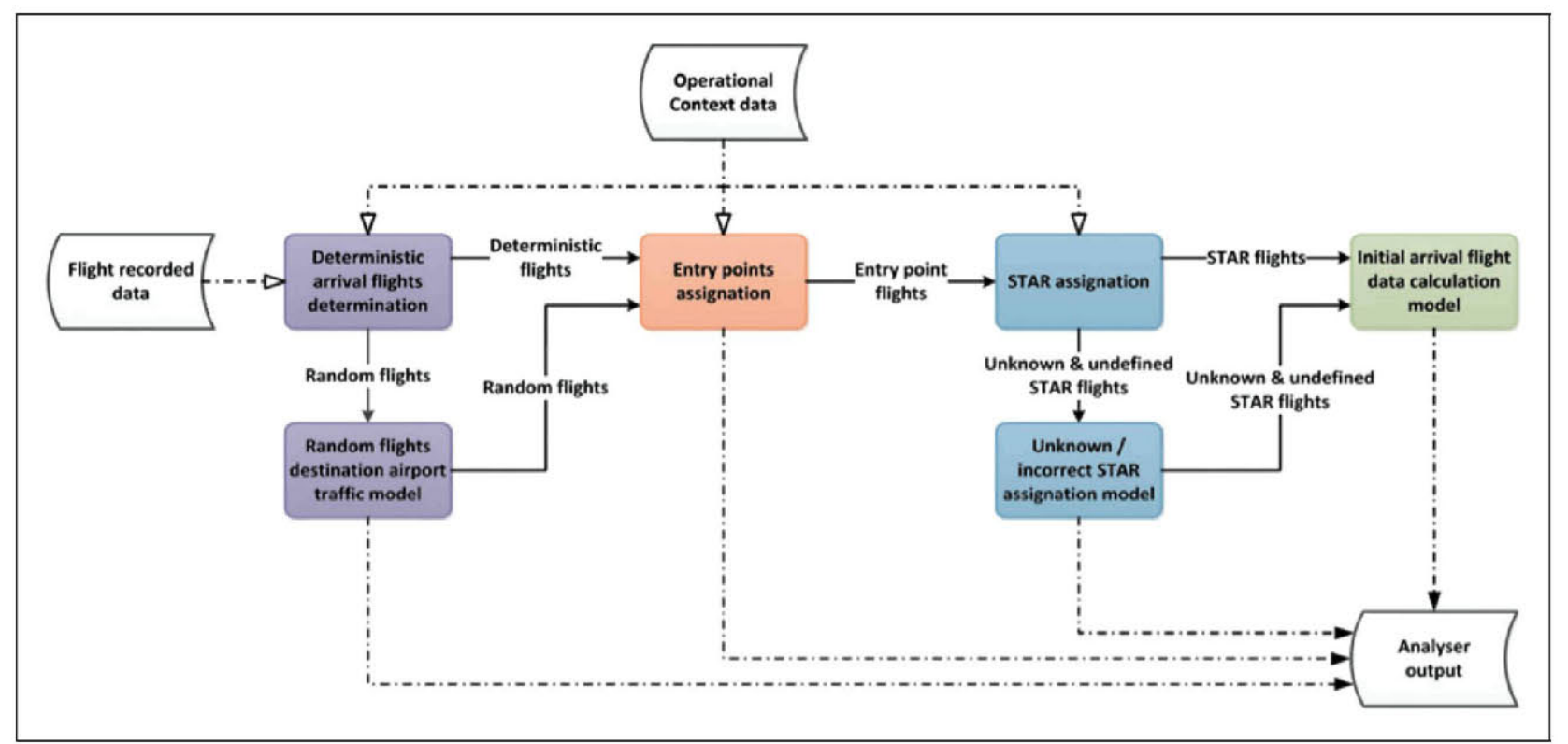

Figure 2. Traffic analyser for arrival aircraft. 
There are four different kinds of flights to be included in the traffic model, depending on origin and destination: (1) arrival flights; (2) departure flights; (3) domestic flights (internal to TMA); and (4) overfly flights (those with origin and destination airports out of the TMA). Slightly different models must be defined for each type, and all those models will be provided in a unique analyser output. The system analyser first determines the type of flight (taking into account origin and destination), and then performs a suitable analysis for all the traffic of a given kind. As an example, the paper details the complete functionality for arrival traffic, depicted in Figure 2. The same kind of philosophy, based on successive segmentation of aircraft types and routes is applied for every other kind of flight.

This function has four stages, related to identification of deterministic/random arrival flight patterns to an airport, assignation of entry point and STAR, and modelling of initial conditions of arrival aircraft.

To estimate the deterministic pattern, the deterministic arrival flights determination function performs a flight identification and expected time of arrival (ETA) correlation over the database information, to detect repetition patterns of flights in the following cases (with a threshold related to a given percentage of occurrences): every day of the week, several days of the week, one day of the week or special days selected by the user. It groups origin airports using a polar grid with respect to TMA centre, so that all airports in a cell of that grid will be equivalent. A logarithmic law is used to define grid limit ranges. In addition, aircraft types for a given deterministic flight follow a discrete random distribution, whose probabilities will be estimated. The information extracted will be a list of deterministic flights, with the following parameters:

- Flight identification

- Origin (grid cell)

- Destination airport

- ETA to the airport

- Repetition pattern

- Aircraft type list. For each aircraft type used in this flight:

- Identifier of the type

- Percentage of arrivals of this deterministic flight with this aircraft type

The random flights destination airport traffic model function obtains the random traffic model for the arrival flights to each airport, as a set of concurrent arrival Poisson processes, parameterised just by the average number of flights per time unit. This process segments the overall traffic in components according to destination airport, origin and aircraft type, and day type, calculating the average number of arrival flights per hour for each component. This is the only parameter needed for the generation of the arrival traffic.
The entry points assignation function finds patterns in the TMA entry points of the flights with a determined origin (grid cell) and destination airport. If there is more than one potential entry point for a given pair entry point-destination airport and aircraft type, it obtains a discrete probability distribution function and its associate parameters (percentages related to each entry point). The output information will be a table with: origin airport (grid cell); destination airport; aircraft type class; and entry point list. For each entry point, it provides its identification and the percentage of arrivals using it (with this origin, destination and aircraft type).

The STAR Assignation function uses the information of all the flights to find patterns in the STAR used by the flights with a determined entry point and destination airport, for each aircraft type class. If there is more than one potential STAR for a given pair entry point-destination airport and aircraft type, the system again obtains a discrete probability distribution function and its associate parameters to model the STAR assignation. So it calculates a table with: entry point; destination airport; aircraft type class; and STAR list. For each STAR, it provides its identification and the percentage of arrivals using it (with this entry point, destination and aircraft type).

The unknown/incorrect STAR assignation model infers routes not considered by the STAR definition. Sometimes, the flights could follow such a route, due to controller vectoring. In that case, this function will define a pseudo-STAR for those routes, and perform an analysis similar to the STAR extraction but taking into account those pseudo-STARs, in addition to the nominal ones. The output table is similar to the previous one, but the identification entry is a pseudoSTAR identification, provided by the analyser. In addition, the pseudo-STAR will be provided as an independent output file to be appended to the operational context and used by the traffic generator as if it were a nominal STAR, defined through a list of associated waypoints or significant points.

Finally, the initial arrival flight data calculation model function is in charge of modelling the arrival flight initial conditions. Each kind of flight (arrival, departure, domestic and overfly) has its own initial data calculation procedure. For arrival traffic, it estimates mean and variance of several probability distribution functions and its corresponding parameters:

- Cruise velocity (corresponding to entry point). It estimates distribution parameters for each origin, destination airport within the TMA and aircraft type.

- Entry point altitude (corresponding to entry fix point). There will be a different distribution for each origin (grid cell), destination airport within the TMA and aircraft type.

- In addition, there are several terms to be calculated to model the time of injection for flight generation: - Landing delay distribution: it is the time difference between the ETA and the actual landing 
time. The analyser calculates distribution parameters per origin airport cell, destination airport, entry point and aircraft type.

- Duration of flight inside the TMA: it is the difference between entry fix point and landing time. Distribution parameters are obtained per STAR and aircraft type.

The previous description just focuses on arrival flights, but in the following the approach used for the other flight types is summarised:

- For departure flights, the deterministic arrival flight determination and the random flights destination airport model extraction functions are substituted by the deterministic departure flight determination and the random flights departure airport model extraction functions, with similar functionalities. Additionally, the entry point assignation function and the STAR assignation function are substituted by the exit point assignation function and the SID assignation function, with an equivalent functionality to that of arrival flights. The undefined and unknown STAR assignation model extraction and the initial departure flight data calculation model function are substituted by the incorrect and unknown SID assignation model extraction function and the initial departure flight data calculation model function, which are also homologous to the arrival flight functions. The most relevant changes are those related to the initial flight data, and specially to mass, based here on BADA models, and on another simplified modeling of the length of the flight, and the different climb, cruise and arrival processes and fuel consumption.

- For domestic flights, internal to TMA, the corresponding functions used are deterministic domestic flights determination function and random domestic flight traffic model function, equivalent to those of arrival flights. Then, there are route assignation and undefined and unknown route assignation model extraction functions, also similar to those of the arrival aircraft and, finally, an initial domestic flight data calculation model function. This function is a simplified version of the one for departure flights, as those very short flights typically do not reach the cruise level.

- For overfly flights, the corresponding initial functions are deterministic overfly flight determination function and random overfly flight model extraction, very similar to those for arrival flights. Moreover, there is an overfly route assignation function and an undefined and unknown overfly route assignation model extraction function, also equivalent to those relevant for arrival flights. Finally, there is an initial overfly flight data calculation model function which, considering the origin and destination airports, makes a guess of the aircraft mass and also calculates some other relevant patterns such as entry or exit point altitudes or velocities, etc.

\section{Detailed functionality of traffic generator}

The traffic generator uses a traffic pattern file (either from traffic analyser or user-edited) and produces a set of synthetic flights statistically consistent with that description.

As the models used and the data needed to generate each kind of flight are different, generation is different for each type of flight. In addition, there is a traffic validation function to test, prior to and after generation, that the operational limits (maximum number of operations per hour in an airport) are not surpassed. Generation will be performed for a contiguous time interval - it could be a set of hours, a complete day (of predefined type with respect to traffic patterns), or even a set of consecutive days (of predefined types with respect to traffic patterns), up to a complete year.

As depicted in Figure 3 the traffic generation starts with an initial traffic validation that checks if the requested traffic pattern is within certain operational limits. Adding all traffic patterns in each operational context element, we will check that airport number of operations per hour, entry point number of operations per hour, exit point number of operations per hour and TMA traffic per hour are not too high from the operational point of view. If any of those thresholds are exceeded or there is an unknown entry point, we will stop generation, and inform about the problematic traffic pattern. As an example, we will describe in this section the part of the traffic generator that creates arrival flights according to the arrival flight traffic patterns. Similar processes are performed for the rest of flight types, with some differences in the models, especially of initial flight data generation.

As can be seen in Figure 4, the generation for arrivals is performed in a set of steps in which we consecutively refine the information regarding individual flights or groups of flights.

Deterministic destination airport selection function generates the deterministic pattern of incoming flights from the traffic pattern file. The traffic generator generates flights consistent with all deterministic patterns for the desired time interval, with the associated ETA and destination airport. This module provides a list of flights including: flight identification; origin (grid cell); destination airport; ETA; and aircraft type.

Afterwards, random flights destination airport generation function generates additional flights according to the random patterns of flights arriving to each airport using the Poisson processes whose parameters were obtained in the analysis phase. It performs a nested loop over destination airports, origin airport grid cells, aircraft type and day type, and for each hour it randomly generates the flights. Again, each 
flight definition has the same parameters as for deterministic flights, except for the flight identification being a random string, and ETA being obtained using the Poisson process.

Next, entry point assignation function generates the information about the TMA entry points of the flights with a determined origin, destination airport and aircraft type. It generates samples of a discrete probability distribution function from its associated parameters (percentages related to each entry point, as derived by the traffic analyser or edited by the user) to generate the TMA entry point assignation.

Then, STAR assignation function defines the STAR used by each flight with a determined entry point, destination airport and aircraft type, using again a discrete probability distribution function and its associate parameters (percentages related to each STAR). In this step, undefined STARs (those which were not in the operational context definition) are equivalent to defined STARs.
Finally, initial arrival flight data generation function generates values for the arrival flight initial conditions. Note that each kind of flight will have its own initialisation procedure. The models next described will be used to generate the needed arrival flight data (in italics).

Cruise velocity and entry point altitude are generated as samples from the analyser-provided distributions for our entry point, provided origin, destination airport within the TMA, and aircraft type are known. Flight injection time in the simulation will be calculated to ensure arrival to runway at a time near the ETA. This time is calculated as ETA + Landing delay-Duration of flight inside the $T M A-D u r a t i o n$ of flight prior to entrance in TMA, where: ETA was previously generated; Landing delay and Duration of flight inside the TMA are calculated as samples of the adequate distribution estimated for origin airport cell, destination airport, and aircraft type, entry point and STAR; and finally Duration

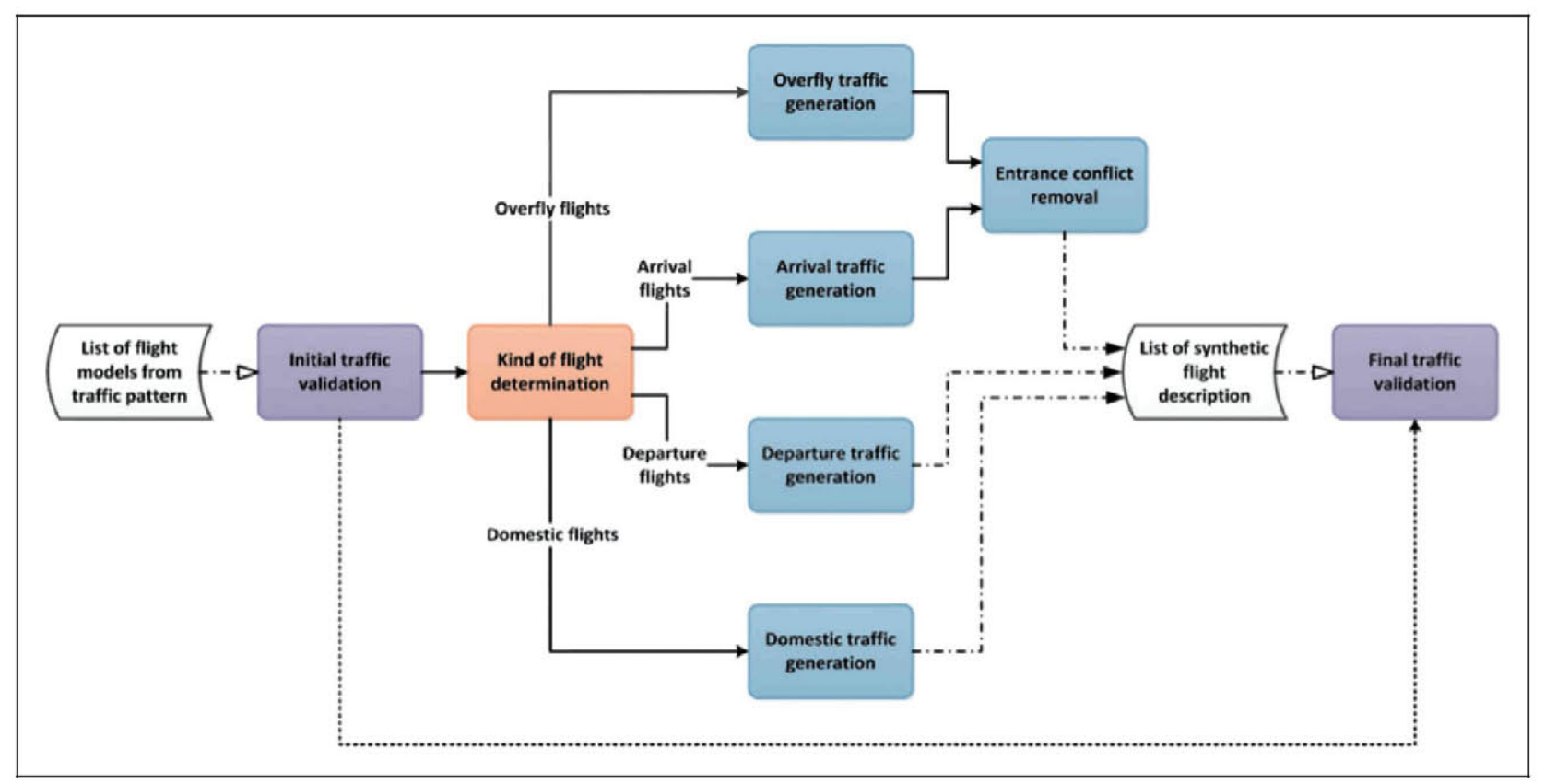

Figure 3. Traffic generator architecture.

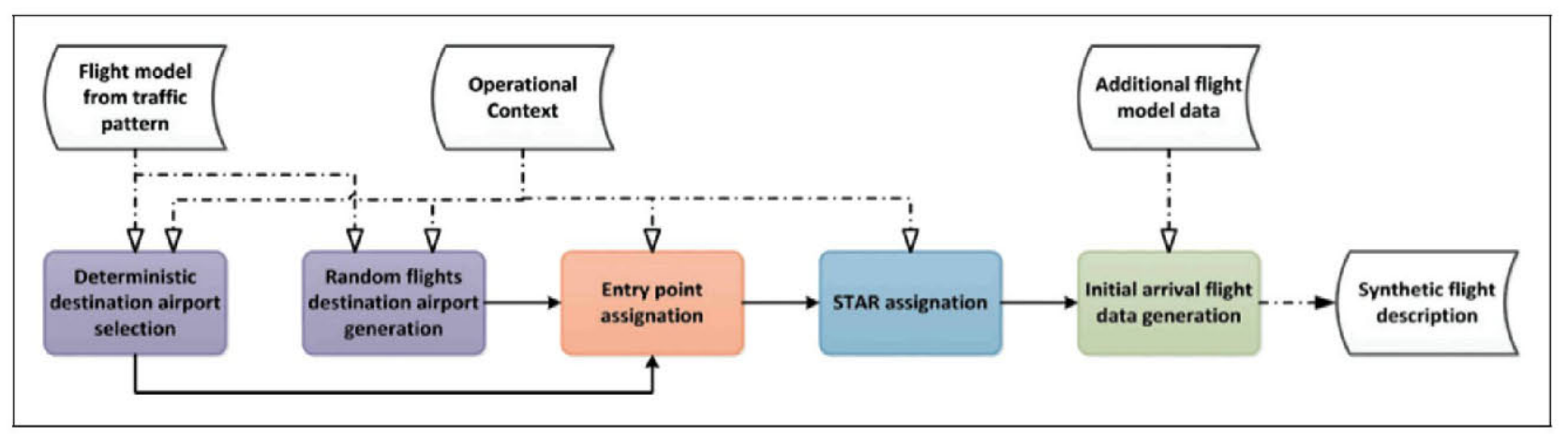

Figure 4. Synthetic traffic generator for arrivals. 
of flight prior to entrance in $T M A$ is a user-defined constant.

The flight initial conditions assume constant height, heading and speed, converging towards the TMA, injected at the previously defined time. The Heading will be calculated assuming the flight comes directly from the centre of the origin cell to the entry point. The Initial position assumes this pattern and predicts back in time from entry point position, using the Duration of flight prior to entrance in TMA constant and the previously generated Cruise velocity.

To perform accurate trajectory predictions, the simulators often needs the aircraft mass at initial position and the performance model to be used. Equation (1) is used to derive the mass from a set of simplified tables, based on the exploitation of $\mathrm{BADA}^{23}$ data (from .PTF file, containing precomputed data). Aircraft Mass, $M_{\text {initial }}$, is calculated as

$$
\begin{aligned}
M_{\text {initial }}= & M_{\text {dest }}(h, d) \\
& +R O F_{\text {Cruise }}(\text { cruise_altitude }) * T_{\text {exc }} \\
& +M_{\text {min }}+r * M_{\text {pyld }}
\end{aligned}
$$

where

- $R O F_{\text {cruise }}(h)$ : cruise rate of fuel consumption at height $h$, with low mass (from BADA, for each aircraft type and typical airspeed values), as we are at the end of the flight.

- cruise_altitude: en route cruise altitude, for aircraft type.

- $T_{\text {exc }}$ : a sample of a normal random variable taking into account remaining fuel for $100 \mathrm{~min}$ and potential increases or decreases of consumption along flight. It will have a mean of 100 and a variance given in an additional flight model definition file.

- $M_{m i n}$ : minimum mass of the aircraft as defined in BADA.

- $M_{p y l d}$ : payload mass of the aircraft as defined in BADA.

- $r$ : a sample of a uniform random variable from Min_Ocupation to Max_Ocupation (defined in additional flight model definition file).

Additionally, $M_{\text {dest }}(h, d)$ is the fuel consumption needed to arrive from an injection point to airport, calculated using equation (2) (based on a table similar to that of .PTF BADA files), depending on $h$ (entry point altitude) and $d$ (distance travelled inside the TMA). First, the function computes the time needed to descend from a given flight level $F L_{i+1}$ to the lower level $F L_{i}$ (defined in BADA .PTF files) using a vertical speed of $R O C D_{i}$ (in BADA model), to be called $T_{\text {desc }}(i)$

$$
T_{\text {desc }}(i)=\frac{F L_{i+1}-F L_{i}}{R O C D_{i}} * 100
$$

The fuel spent to descend from height $h$ to airport level (assumed to be zero) can be roughly estimated, knowing the rate of fuel in descent for each level as provided by BADA $\left(R O F_{\text {descend }}(i)\right)$ as

$$
M_{\text {desc } \_n o m}(h)=\sum_{i=o}^{F L(h)} R O F_{d e s c e n d}(i) * T_{\text {desc }}(i)
$$

If we assume the aircraft descending at the nominal true airspeed VTAS descend $(i)$ (from BADA) between $F L_{i+1}$ and $F L_{i}$, the traversed distance during the complete nominal descend is

$$
d_{\text {desc_nom }}(h)=\sum_{i=0}^{h} V T A S_{\text {descend }}(i) * T_{\text {desc }}(i)
$$

So, assuming prior to descend the aircraft is at level flight at the nominal true airspeed $\left(V T A S_{\text {cruise }}(h)\right)$, and $T_{\text {prev }}$ is the duration of flight prior to entrance in TMA (user-defined time constant), the complete fuel consumption mass is

$$
\begin{aligned}
M_{\text {dest }}(h, d)= & \text { OOF }_{\text {Cruise }, \text { low }}(h) \\
& *\left(T_{\text {prev }}+\frac{d-d_{\text {desc_nom }}(h)}{V T A S_{\text {cruise }}(h)}\right) \\
& +M_{\text {desc_nom }}(h)
\end{aligned}
$$

Similar models for the rest of the types of flights might be derived using available BADA data. Specifically:

- For departure flights, the deterministic designation airport selection and the random flights destination airport model generation functions are substituted by the deterministic departure airport selection and the random flights departure airport model generation functions, with similar functionalities. The deterministic and probabilistic entry point assignation functions and the deterministic and probabilistic STAR assignation functions are also substituted by the deterministic and probabilistic exit point assignation functions and the deterministic and probabilistic SID assignation functions. The initial arrival flight data calculation model function is substituted by the initial departure flight data calculation model function.

- For domestic flights, internal to TMA, there is a set of functions - deterministic domestic flight selection function and random domestic flights model generation function, deterministic route assignation function, probabilistic route assignation model extraction function, and initial domestic flight data generation function - which are equivalent to arrival flight functions.

- For overfly flights, the system has deterministic overfly flight selection, random overfly flight model generation, deterministic overfly route assignation, probabilistic overfly route assignation model and initial overfly flight data generation functions. 
For departure, internal and overfly flights, the corresponding modifications to the fuel consumption functions are also implemented. Two final processes remain to complete the generation of traffic, depicted in Figure 3. Firstly, the entrance conflict removal function tests if there is a potential conflict at any entry point after aggregating arrival and overfly flights, modifying the injection times of conflict aircrafts so that they are separated.

Finally, the final traffic validation function rechecks if the generated traffic pattern is within the operational limits defined by the user, giving an error if any of the operational limits is not respected. A user modifiable $10 \%$ additional guard is set, so that even not completely compliant traffic (slightly higher traffic than the one approved for the aerodrome, point or sector) is allowed. These limits will be checked against operational limits: airport number of operations per hour; entry point number of operations per hour; exit point number of operations per hour; and TMA traffic per hour.

\section{Traffic analyser and generator HMI}

In this section, we present the main aspects of the HMIs of both traffic analyser and traffic generator. Special emphasis is given to output HMIs owing to the fact that they are the means to be used by the operator to either understand the traffic situation or to validate the generated scenario.

The main menu of the synthetic traffic generator allows selecting the desired functionality of the program (traffic analyser input HMI, synthetic traffic generator input HMI, analyser output HMI and generator output HMI).

\section{Traffic analyser HMI}

Traffic analyser input HMI allows the selection of XML files including traffic data, operational context definition, aircraft type and aircraft type class definition, and to edit or select a user preference file. Figure 5 shows this HMI. Using this window, the user can select or edit the corresponding configuration files in order to execute the analyser. When the window comes up for the first time, a default file path is loaded for each configuration file, unless the default file does not exist. In that case, the corresponding text box will be empty. The user can browse files, edit them, import them (using standard BADA or AIXM formats), and finally execute the traffic analyser (big 'Analyse' button) or return to the main menu.

Traffic pattern analyser output HMI is devoted to the presentation of the results of the analysis module. There are means for the representation of aggregated data and pattern-related data. After selecting the output files to be presented (traffic pattern obtained from the analyser, operational context, pseudo-routes and user preferences), the analyser output HMI offers the user the analysis results in three different formats: traffic maps, number of flights and additional traffic data (table of flights). Figure 6 shows a capture of the three selection windows in the same order as they appear after file selection, enquiring the user firstly about the kind of visualisation, secondly about the type of flight pattern and, finally, about the type of traffic statistic.

In a traffic map the user selects a time interval and a time increment, and the software shows, using a colour code, the number of aircraft passing through each leg for each time interval (aggregated taking into consideration the adequate flight types and adding deterministic flights and the mean number of random flights). This is shown in Figure 7, which represents the arrival traffic map of Gran Canaria, for normal days averaged by hour, rebuilt from a synthetic data sample. The traffic in this sample just

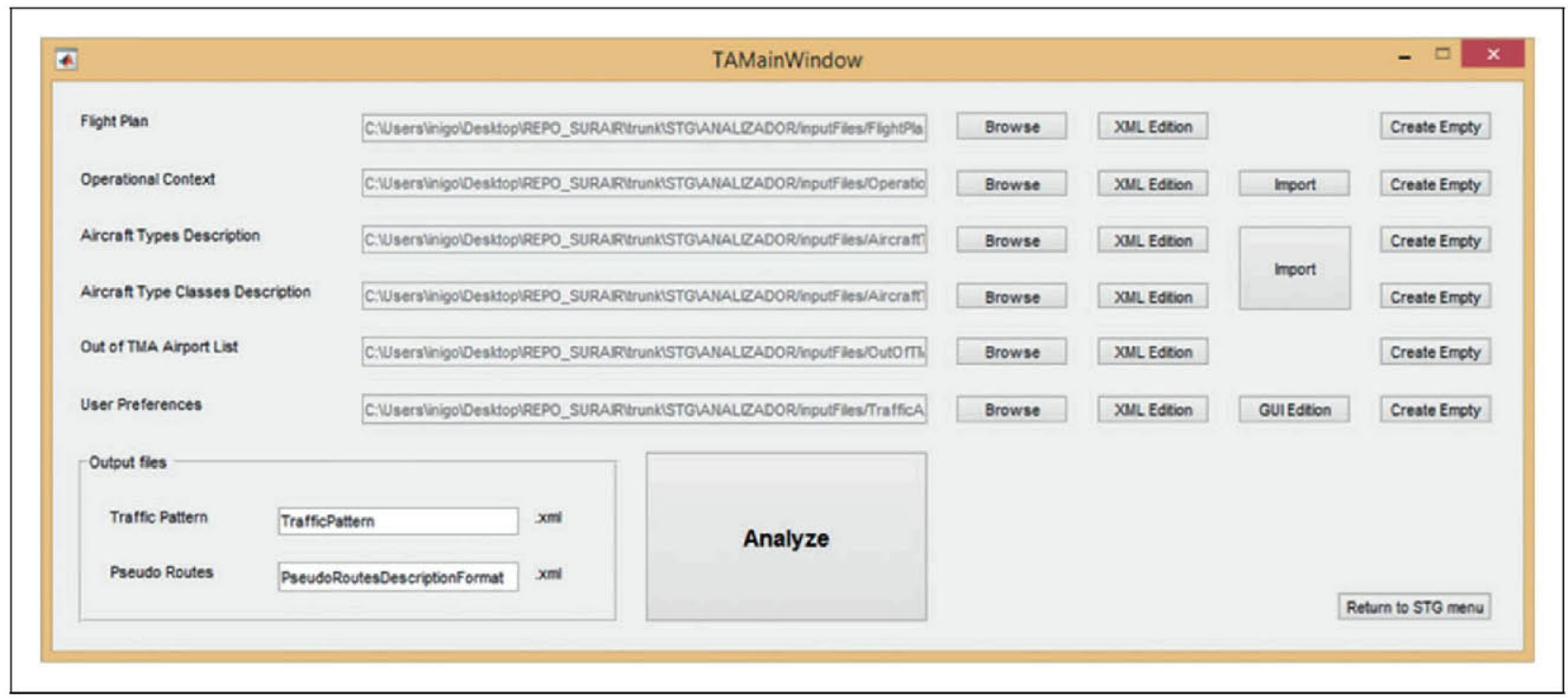

Figure 5. Traffic analyser initial window. 
covered SAMAR4C, TERTO6C/TERTO6Z and RUSIK4C/RUSIC4Z STARs, and there is no traffic following a random route in this sample.

Meanwhile, for the Number of Flights plot, the user selects firstly a scenario element (STAR, SIR, leg, airport, etc.), and secondly a time interval to be averaged, as seen in Figure 8 (a), obtaining a plot with the evolution of number of aircraft versus time (per hour) for the selected element, as depicted in Figure 8 (b).

\section{Traffic generator HMI}

Initially, from the main menu the user can select the files needed to generate traffic results. The traffic generator input window is very similar to that of the analyser (Figure 5), except for the necessary files to be selected and the main button, which now reads 'Generate' instead of 'Analyse,' as the objective of this HMI is to control the generation part of our system.

The traffic generator also has an output HMI, which may be used by an expert to perform a visual validation, comparing the definition of the traffic patterns and the finally obtained synthetic traffic (maps with traffic statistics, histograms, etc.). The HMI is functionally equivalent to that of the analyser, enabling the inspection of aggregated traffic data or providing a table/list with generated flights. All representations are derived from synthetic flights, not from generator inputs. Again, traffic maps and number of flight diagrams may be depicted, with selection tools (for specific airspace/hour/type of day), similar to those available for the traffic analyser.

\section{Conclusions and future work}

In this paper, we have summarised the design of a prototype traffic analysis and generation tool. This system can derive meaningful data on TMA traffic (traffic patterns) and generate consistent traffic samples which may be able to help in traffic simulation with a much richer and complete amount of data, with bigger controlled variability than that in current systems and with a lower cost to implement simulation scenarios. The described system enables a much improved understanding by the ATM engineer of the traffic history, and simplifies the definition of WHATIF scenarios, enabling the assessment of performance

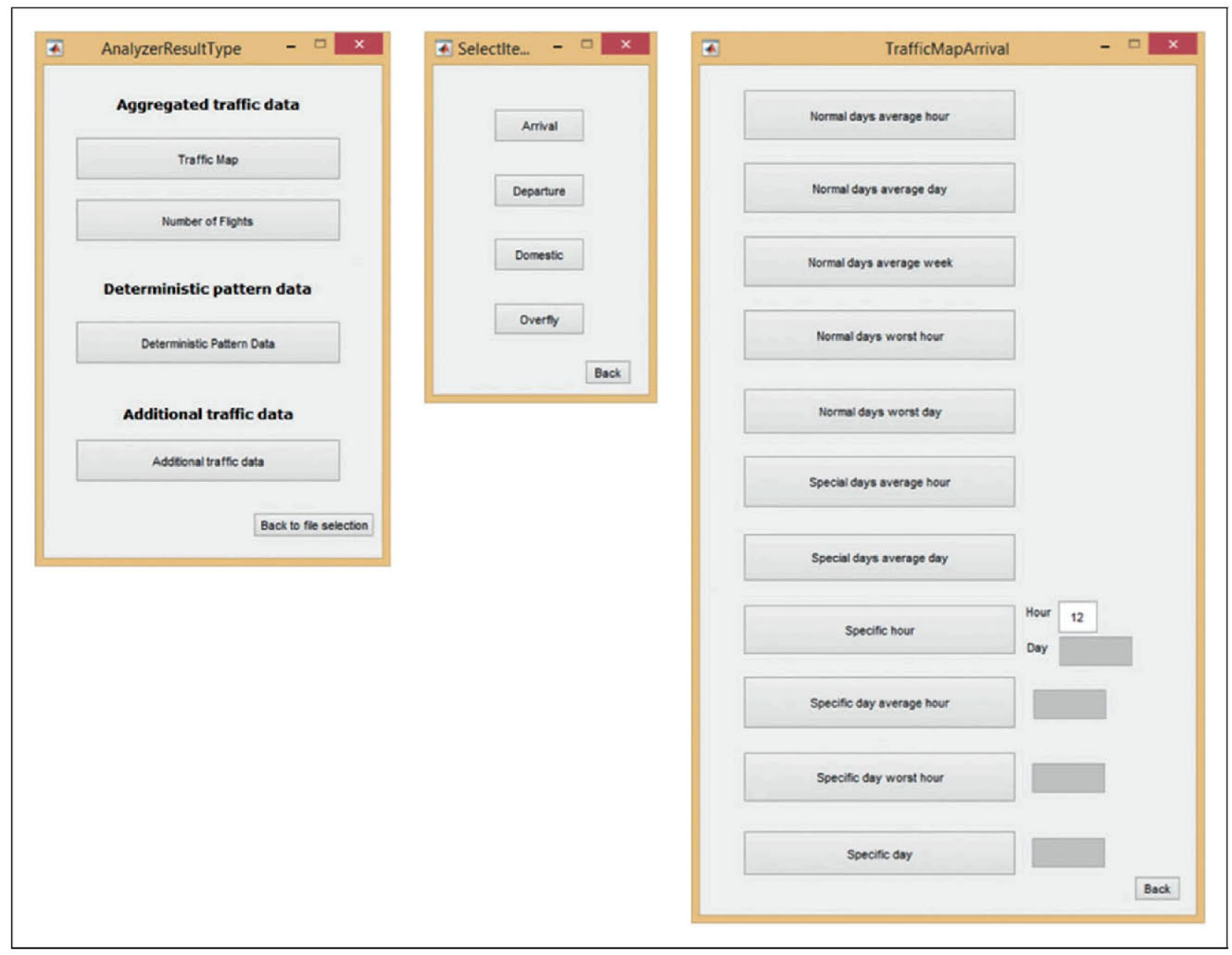

Figure 6. Selection of traffic map representation. 


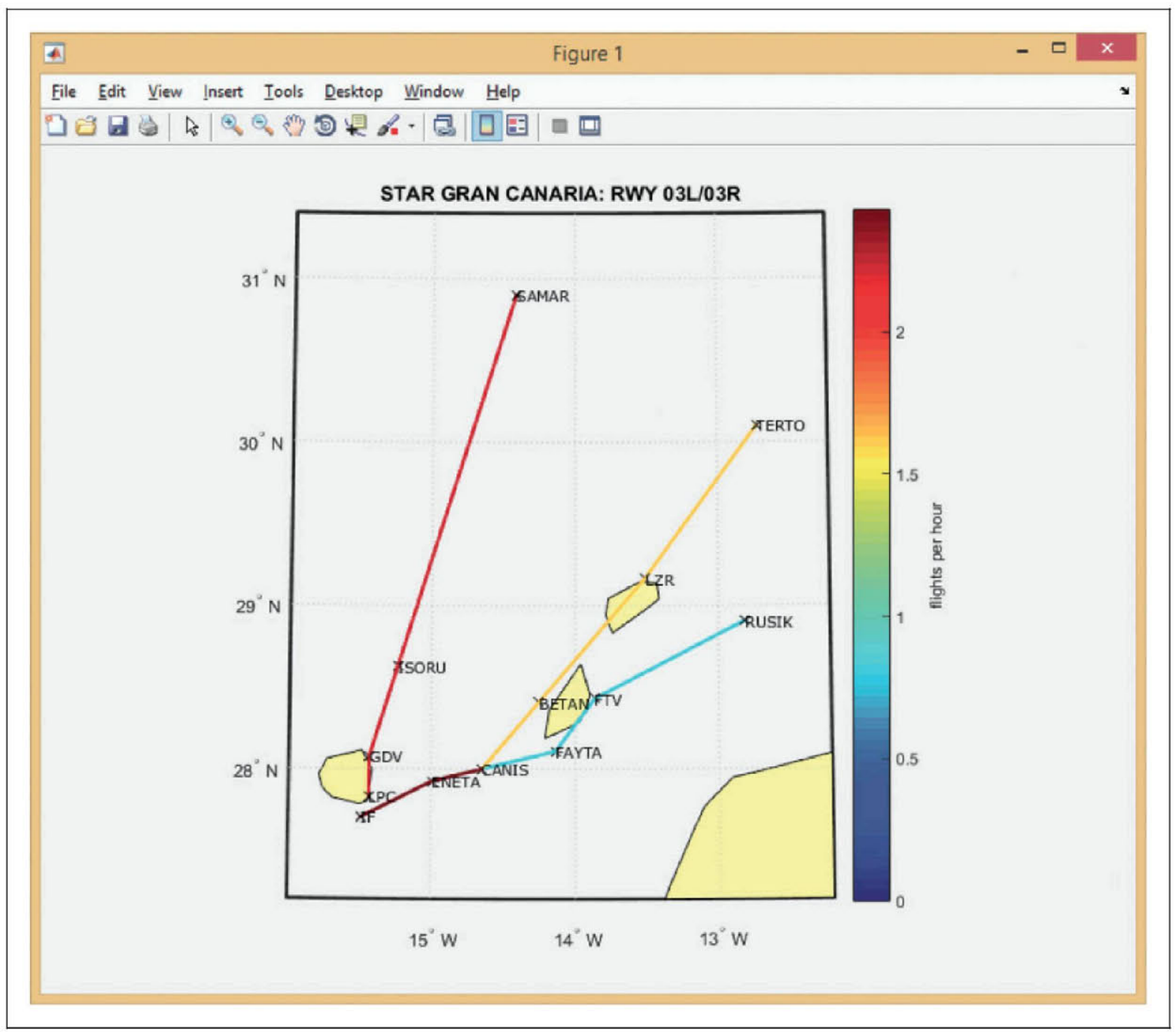

Figure 7. Example of analyser result presentation.

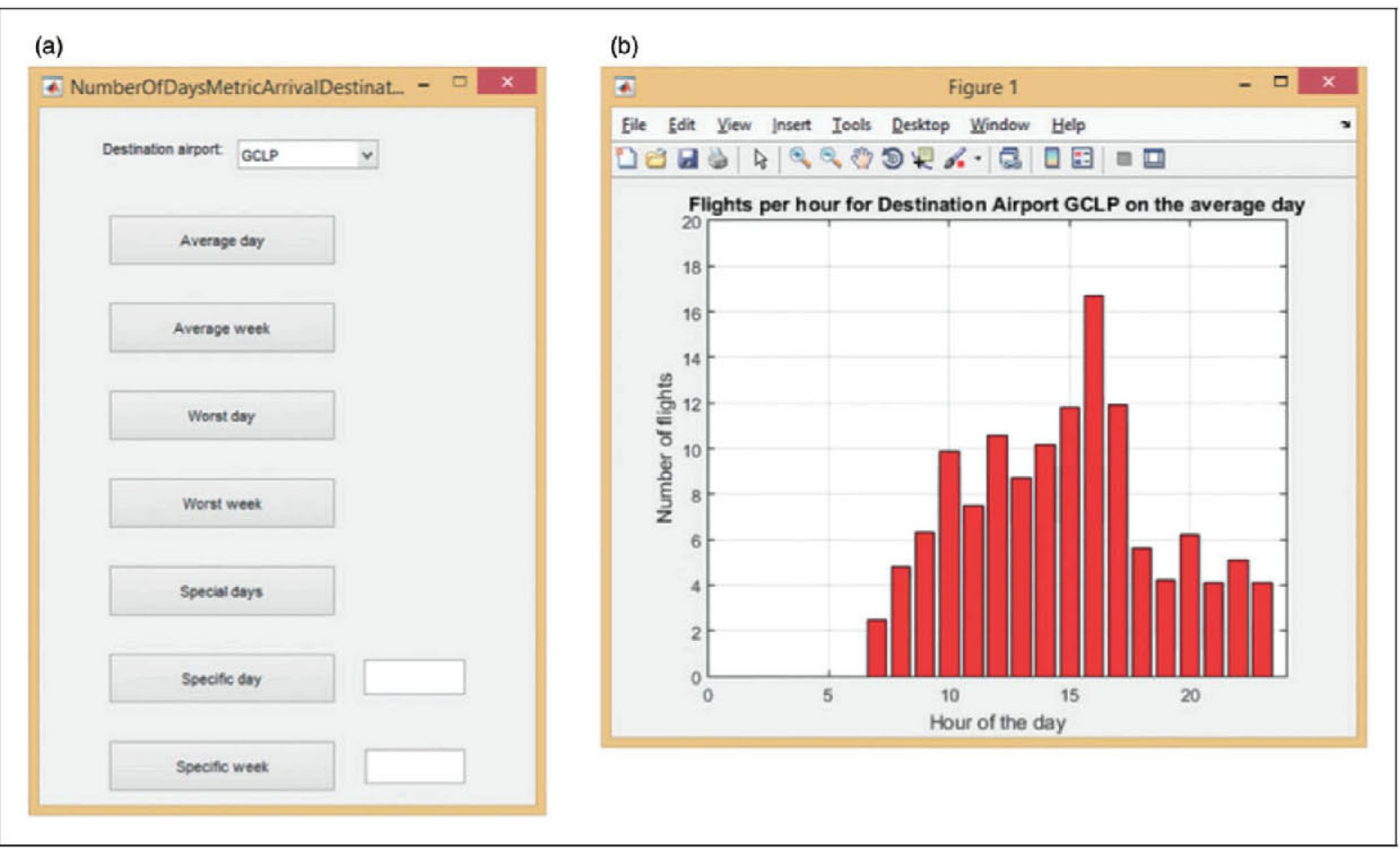

Figure 8. Number of flights selection (a) and representation (b). 
modifications due to traffic changes, the training under new traffic conditions, etc. Future developments of this tool include extending this philosophy to new airspace areas (i.e. en-route, oceanic), and the analysis of potential correlations of some of the traffic patterns data and especially of the effects of meteorological conditions both in the TMA and in the surrounding areas, which may impact airport configurations, delays, traffic congestion, etc.

It should also be acknowledged that there is much effort needed to complete the research with respect to validation of the system with real traffic, and with final users. Especially important here is the need to make the users aware of the quality of the provided traffic in terms of consistency with real traffic. The more the traffic (in stationary conditions) available, the higher will be the quality of models, but at the same time the higher granularity will be available to the extraction of deterministic and random traffic patterns. Some rare events with small data samples may keep rare events with bigger samples or become actual patterns with low associated traffic. Those small components of traffic typically are of lower importance in the complete system outcome in terms of delay, throughput, etc. but may be relevant for some other ATM aspects.

\section{Funding}

This work is a result of the continuous collaboration between Indra and UPM both in ATLANTIDA project and SESAR programme. The research has also been partially funded by the UPM's project "Tecnologías Avanzadas para lo Monitorización y Gestión Remota del Tráfico Aéreo de Vehículos Pilotados y no Pilotados (RP1509550C02)" and by Spanish Ministry of Economy and Competitiveness through grant TEC2014-57022-C2-1-R.

\section{Conflict of interest}

The authors declared no potential conflicts of interest with respect to the research, authorship, and/or publication of this article.

\section{References}

1. Domino DA, Tuomey D, Stassen HP, et al. Paired approaches to closely spaced runways: Results of pilot and ATC simulation. In: $33^{\text {rd }}$ IEEE/AIAA digital avionics systems conference (DASC), Prague, Czech Republic, 2014, pp.1B2-1-1B2-15.

2. Penhallegon WJ and Bone RS. Flight deck-based merging and spacing impact on flight crew operations during continuous descent arrivals and approaches. In: $27^{\text {th }}$ IEEE/AIAA digital avionics systems conference, St. Paul, MN, USA, 2008, pp.3.C.3-1-3.C.3-14.

3. Ho N, Johnson W, Arutyunov V, et al. Design and evaluation of NextGen aircraft separation assurance concepts. In: $31^{\text {st }}$ IEEE/AIAA digital avionics systems conference (DASC), Williamsburg, VA, USA, 2012, pp.4D1-1-4D1-12.

4. Alliot J-M, Granger G and Pomeret J-M. Arithmetic simulation and performance metrics [ATC]. In:
Proceedings of $21^{\text {st }}$ digital avionics systems conference, Irvine, CA, USA, 2002, pp.2D2-1-2D2-6.

5. Mohleji SC and Hoffman J. Performance analysis of North Pacific Operations using an automated air traffic control system simulation. IEEE Trans Control Syst Technol 1993; 1: 179-185.

6. Calderon-Meza G, Sherry L and Consiglio M. Multiagent simulation of NAS infrastructure utilization in the presence of airline policies. In: Integrated communications, navigation and surveillance conference, Arlington, VA, USA, 2009, pp.1-7.

7. Sislak D, Volf P, Kopriva S, et al. Agentfly: NAS-wide simulation framework integrating algorithms for automated collision avoidance. In: Integrated communications, navigation and surveillance conference (ICNS), Herndon, VA, USA, 2011, pp.F7-1-F7-11.

8. Hughes $\mathrm{KK}, \mathrm{Wu} \mathrm{CY}$, Lamp JL, et al. Optimizing airport arrival operations: A systems engineering analysis of Washington Dulles International Airport. In: 2012 IEEE systems and information design symposium (SIEDS), Charlottesville, VA, USA, 2012, pp.145-149.

9. Tofukuji N. An enroute ATC simulation experiment for sector capacity estimation. IEEE Trans Control Syst Technol 1993; 1: 138-143.

10. Ferrante $\mathrm{J}$ and Johnson C. Balancing Air Traffic Control workload across dual area navigation arrival procedures. In: Integrated communications, navigation and surveillance conference, Arlington, VA, USA, 2009, pp.1-11.

11. Teutsch $\mathbf{J}$ and Hoffman E. Aircraft in the future ATM system-exploiting the 4D aircraft trajectory. In: $23^{\text {rd }}$ Digital avionics systems conference, Salt Lake City, UT, USA, 2004, pp.3.B.2-31-22.

12. Miller ME, Capozzi B, Guzhva V, et al. Airborne execution of flow strategies simulation. In: $33^{\text {rd }}$ IEEE/ AIAA digital avionics systems conference (DASC), Colorado Springs, CO, USA, 2014, pp.1D1-1-1D1-6.

13. SESAR | Partnering for smarter aviation, http:// www.sesarju.eu/ (accessed 10 March 2015).

14. Next Generation Air Transportation System (NextGen), https://www.faa.gov/nextgen/

15. Fabry JM and Lupinetti AA. Applications of simulation and artificial intelligence technology for ATC training. Proc IEEE 1989; 77: 1762-1765.

16. Scardina JA, Ryberg P-Y and Gerstenfeld A. Future ATC automation aids based upon AI technology. Proc IEEE 1989; 77: 1625-1633.

17. Kornecki AJ, Hilburn TB, Diefenbach TW, et al. Intelligent tutoring issues for air traffic control training. IEEE Trans Control Syst Technol 1993; 1: 204-211.

18. Mo S, Li H, He X, et al. Development of the ATC Simulators. In: 16th international conference on artificial reality and telexistence-workshops, ICAT '06, Hangzhou, China, 2006, pp.333-338.

19. Johnson DR, Nenov VI and Espinoza G. Automatic speech semantic recognition and verification in air traffic control. In: $32^{\text {nd }}$ IEEE/AIAA digital avionics systems conference (DASC), East Syracuse, NY, USA, 2013, pp.5B5-1-5B5-14.

20. Gillet S, Nuic A and Mouillet V. Enhancement in realism of ATC simulations by improving aircraft behaviour models. In: $29^{\text {th }}$ IEEE/AIAA digital avionics systems conference (DASC), Salt Lake City, UT, USA, 2010, pp.2.D.4-1-2.D.4-13. 
21. Volf P, Jakubüv J, Koranda L, et al. Validation of an air-traffic controller behavioral model for fast time simulation. In: Integrated communications, navigation and surveillance conference (ICNS), Herndon, VA, USA, 2014, pp.T1-1-T1-9.

22. Oaks R, Liu S, Zhou D, et al. Methodology for the generation of air traffic scenarios based on recorded traffic data. In: $22^{\text {nd }}$ digital avionics systems conference, DASC 03, Indianapolis, IN, USA, 2003, pp.5.C.3-5.1-7.

23. Base of Aircraft Data (BADA)| Eurocontrol [Internet], https://www.eurocontrol.int/services/bada (accessed 10 March 2015).
24. Besada JA, Frontera G, Crespo J, et al. Automated aircraft trajectory prediction based on formal intent-related language processing. IEEE Trans Intell Transp Syst 2013; 14: 1067-1082.

25. Besada JA, Portillo J, de Miguel G, et al. Traffic analysis and synthetic scenario generation for ATM operational concepts evaluation. In: $28^{\text {th }} I E E E / A I A A$ digital avionics systems conference, DASC '09, Orlando, FL, USA, 2009, pp.2.A.1-1-2.A.1-14. 